\title{
Apresentação clínica da doença de Chagas crônica em indivíduos idosos
}

\section{Clinical presentation of chronic Chagas disease in elderly individuals}

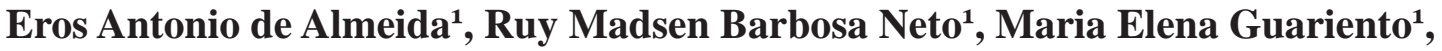 \\ Jamiro da Silva Wanderley ${ }^{1}$ e Milton Lopes de Souza ${ }^{1}$
}

\begin{abstract}
RESUMO
Com o objetivo de avaliar a apresentação clínica da doença de Chagas em idosos foi realizado estudo retrospectivo utilizando-se os prontuários de doentes atendidos em ambulatório de referência. A casuística foi dividida em idosos ( $\geq 60$ anos) e não idosos. Avaliou-se: sexo, co-morbidades, forma clínica, eletrocardiograma e títulos das sorologias. Idosos (61 casos): média de idade de 66,0 \pm 5 anos, 67,2\% do sexo feminino; comorbidades em 59\%, mais freqüente a hipertensão arterial sistêmica $(H A S)=39,3 \%$; forma indeterminada $=1,6 \%$, forma cardíaca $=88,5 \%$, forma digestiva $=36,1 \%$; alterações freqüentes no eletrocardiograma: bloqueio divisional ântero-superior esquerdo $(B D A S E)=41 \%$, bloqueio completo de ramo direito $(B C R D)=32,8 \%$, extra-sístole ventricular $(E V)=22,9 \%$. Não idosos (61 casos): média de idade: $39,30 \pm 8,36$ anos, 54,1\% do sexo feminino; comorbidades em 50,8\%, mais freqüente a HAS (26,2\%); forma indeterminada= $18 \%$ $(p<0,05)$, forma cardiaca $=78,7 \%$, forma digestiva $=32,8 \%$; alterações freqüentes no eletrocardiograma: $B D A S E=24,6 \%, B C R D=21,3 \%, E V$ $=18 \%$. Concluindo, não houve diferenças clínicas entre indivíduos idosos e não idosos e a forma indeterminada predominou nos indivíduos abaixo dos 60 anos.
\end{abstract}

Palavras-chaves: Doença de Chagas. Tripanossomíase americana. Idoso. Forma indeterminada. Eletrocardiograma.

\section{ABSTRACT}

This study had the aim of evaluating the clinical presentation of chronic Chagas disease among the elderly. It was a retrospective analysis of clinical records at an outpatient referral service. The sample was divided into two groups: elderly ( $\geq 60$ years old) and non-elderly. Sex, comorbidities, clinical form, electrocardiogram and serological titers were evaluated. In the elderly group (61 cases), the mean age was $66.03 \pm 5$ years; $67.2 \%$ were female; $59 \%$ presented comorbidities (most frequently systemic arterial hypertension, in 39.3\%); 1.6\% had the indeterminate clinical form, $88.5 \%$ the cardiac form and 36\% the digestive form; and abnormalities were frequently found on electrocardiograms: $41 \%$ presented anterosuperior left bundle branch block (AS-LBBB), 32.8\% presented right bundle branch block (RBBB) and 22.9\% presented ventricular ectopic beats (VEB). In the non-elderly group (61 cases), the mean age was $39.30 \pm 8.36$ years; $54.1 \%$ were female; $50.8 \%$ presented comorbidities (most frequently systemic arterial hypertension, in 26.2\%); $18 \%$ had the indeterminate clinical form ( $p<0.05)$, 78.7\% the cardiac form and $32.8 \%$ the digestive form; and abnormalities were frequently found on electrocardiogram: 24.6\% presented AS-LBBB, 21.3\% RBBB and 18\% VEB. It was concluded that there were no clinical differences between elderly and non-elderly Chagas patients. The indeterminate clinical form predominated in patients less than 60 years old.

Key-words: Chagas disease. American trypanosomiasis. Elderly person. Indeterminate form. Electrocardiogram.

A doença de Chaga é uma doença parasitária causada por um protozoário flagelado: Trypanosoma cruzi. A transmissão vetorial se dá por insetos hematófagos da família Reduviide e subfamília Triatomine, sendo os mamíferos os hospedeiros definitivos e principais reservatórios, atualmente estando controlado no Brasil a principal espécie: Triatoma infestans ${ }^{22}$. Porém, outros mecanismos de transmissão existem como a transfusão sanguínea, vertical e oral, os quais podem originar novos casos da doença de Chagas $^{25}$. A Tripanossomíase americana, descoberta por
Carlos Chagas em $1909^{10}$, constitui ainda hoje uma endemia preocupante uma vez que 16 a 18 milhões de indivíduos podem estar acometidos na América Latina, sendo que no Brasil estima-se entre 1,8 a 2,4 milhões aqueles na fase crônica da doença ${ }^{128}$.

Progressos nos programas de controle, tanto da transmissão vetorial como da transfusional ${ }^{14}$ tornaram raros os casos de formas agudas da doença de Chagas, determinando assim uma elevação da média de idade dos doentes constatada em estudos clínicos $^{19}$ e em candidatos a doação de sangue com sorologia

1. Grupo de Estudos em doença de Chagas, Faculdade de Ciências Médicas, Universidade Estadual de Campinas, Campinas, SP. Endereço para correspondência: Dr. Eros Antônio de Almeida. R. Jorge Nogueira Ferraz 14/74, Jardim Chapadão, $13070-120$ Campinas, SP. Tel: 55 19 3521-7803, Fax: $55193289-4107$

e-mail: eros@fcm.unicamp.br

Recebido para publicação em 6/10/2005

Aceito em 26/4/2007 
positiva para doença de Chagas $^{27}$. Avaliação em censos do governo federal brasileiro ${ }^{18}$ tem demonstrado que a população idosa aumentou e, conseqüentemente, a morbidade em geral vem acompanhando tal situação, não fugindo à regra as doenças infecciosas crônicas, como a de Chagas. Estudos mostram o caráter evolutivo e imprevisível da doença de Chagas para a forma crônica cardíaca demonstrando que esta pode ocorrer em indivíduos idosos ${ }^{213}{ }^{15}$. No entanto, têm sido salientado que as formas graves da doença de Chagas crônica e a evolução desfavorável ocorrem, predominantemente, em indivíduos mais jovens, implicando em incapacidade, aposentadoria precoce e morte antes de se atingir a velhice ${ }^{482} 23$. Esta impressão encontrada na literatura médica sobre 0 assunto pode interferir no diagnóstico da doença de Chagas nesta faixa etária, sendo o quadro clínico sugestivo da doença em idoso atribuído a outras patologias de maior prevalência nestes indivíduos, tais como cardiomiopatia dilatada, cardiopatia isquêmica, cardiopatia por hipertensão arterial, neoplasias do esôfago, etc ${ }^{9}$. Embora comorbidades possam ocorrer, já se demonstrou que a necropsia de indivíduos com mais de 60 anos e com sorologia positiva para doença de Chagas revelou cardiopatia crônica de intensidades variáveis, responsabilizada por distúrbios do ritmo cardíaco e óbito destes doentes ${ }^{21}$.

0 presente estudo teve 0 objetivo de avaliar a apresentação clínica da doença de Chagas em idosos, uma vez que esta faixa etária deve ser considerada em relação a estes aspectos, posto que a atenção médica e social aos chagásicos crônicos é um dos principais desafios dessa enfermidade no Brasil ${ }^{14} 24$.

\section{MATERIAL E MÉTODOS}

0 estudo foi retrospectivo, utilizando-se os prontuários clínicos de doentes do ambulatório do Grupo de Estudos em Doença de Chagas (GEDoCh) do Hospital de Clínicas da Universidade Estadual de Campinas (UNICAMP), onde são atendidos indivíduos adultos, com sorologia positiva. 0 período de avaliação compreendeu os doentes atendidos nos últimos 14 anos. Foram analisados os prontuários em que havia, no mínimo, duas sorologias positiva para doença de Chagas (fixação do complemento e imunofluorescência indireta). Os indivíduos idosos foram selecionados levando-se em consideração os dados do Instituto Brasileiro de Geografia e Estatística ${ }^{18}$, que limita esta faixa etária a maior de 60 anos (Grupo 1). Para comparação utilizaram-se os dados dos prontuários de adultos, também com duas sorologias positivas para Chagas (fixação do complemento e imunofluorescência indireta), com idade inferior a 60 anos (Grupo 2). As seguintes variáveis foram avaliadas: sexo, forma clínica da doença, comorbidades, eletrocardiograma e títulos das sorologias. As formas clínicas em que a doença de Chagas é classificada no GEDoCh são: indeterminada (ausência de sintomas, eletrocardiograma normal, radiografias do tórax e tubo digestivo normais), cardíaca (alterações eletrocardiográficas tais como distúrbios do ritmo, bloqueios na condução do estímulo elétrico cardíaco ou radiografia do tórax com cardiomegalia) e digestiva (esôfagograma ou enema opaco compatíveis com megaesôfago ou megacolon). As variáveis foram comparadas entre os dois grupos, utilizando-se teste não paramétrico qui-quadrado, sendo a significância estatística menor ou igual a 0,05 .

\section{RESULTADOS}

0 grupo 1 foi constituído por 61 indivíduos com idade entre 60 e 84 anos (média de 66,03 $\pm 5,00$ ). 0 grupo 2, com 61 indivíduos com idade entre 22 e 56 anos (média de 39,30 $\pm 8,36$ ).

Os dados relativos às diferentes variáveis estudadas podem ser observados na Tabela 1 e as alterações eletrocardiográficas na Tabela 2.

Tabela 1- Resultados das variáveis estudadas: sexo, forma clínica, eletrocardiograma e comorbidade.

\begin{tabular}{|c|c|c|c|c|}
\hline \multirow[t]{2}{*}{ Variáveis/grupos } & \multicolumn{2}{|c|}{ Grupo 1} & \multicolumn{2}{|c|}{ Grupo 2} \\
\hline & $\mathrm{n}^{0}$ & $\%$ & $\mathrm{n}^{0}$ & $\%$ \\
\hline \multicolumn{5}{|l|}{ Sexo } \\
\hline feminino & 41 & 67,2 & 33 & 54,1 \\
\hline \multicolumn{5}{|l|}{ Forma clínica } \\
\hline indeterminada & 01 & 1,6 & 11 & 18 \\
\hline digestiva & 22 & 36,0 & 20 & 32,8 \\
\hline cardíaca & 54 & 88,5 & 48 & 76,7 \\
\hline Comorbidade & 36 & 59,0 & 31 & 50,8 \\
\hline \multicolumn{5}{|l|}{ Eletrocardiograma } \\
\hline alterado & 52 & 85,2 & 41 & 67,2 \\
\hline
\end{tabular}

Tabela 2 - Freqüiência de alterações eletrocardiográficas nos indivíduos dos grupos 1 e 2.

\begin{tabular}{lrr}
\hline Alterações/Grupos & Grupo 1 (\%) & Grupo 2 (\%) \\
\hline BDASE & 41 & 24,6 \\
BRD & 32,8 & 21,3 \\
EV & 22,9 & 18,0 \\
Alteração primária de repolarização & 11,5 & 11,5 \\
SVE & 11,5 & 4,9 \\
BAV & 8,2 & 13,1 \\
FA & 3,3 & - \\
BRE & 3,3 & 1,6 \\
\hline
\end{tabular}

BDASE: bloqueio divisional ântero superior esquerdo; BRD: bloqueio completo de ramo direito; EV: extrassístole ventricular; SVE: sobrecarga ventricular esquerda; BAV: bloqueio átrio ventricular; FA: fibrilação atrial; BRE: bloqueio completo de ramo esquerdo $(\mathrm{p}>0,05)$.

Em relação ao sexo, houve $67,2 \%$ de mulheres no grupo dos idosos e $54,1 \%$ de mulheres no grupo dos não idosos (Figura 1). Não houve diferença estatística entre os dois grupos.

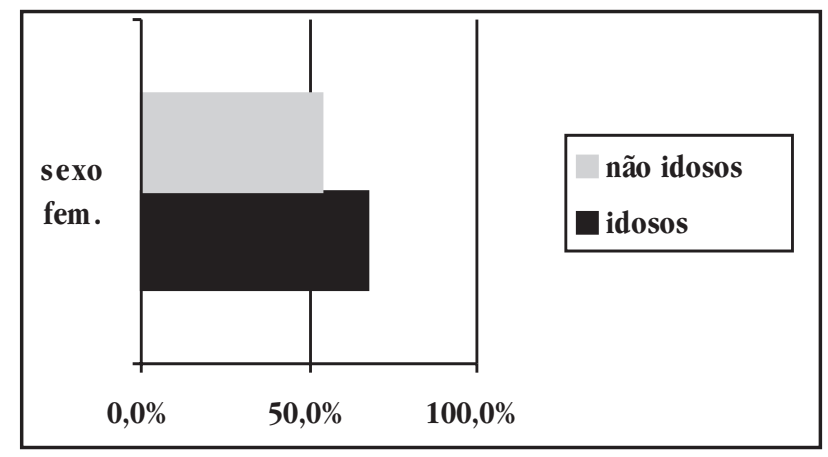

Figura 1 - Distribuição por sexo dos indivíduos dos grupos 1 e 2 (p>0,05). 
As comorbidades ocorreram em $59 \%$ dos idosos e em $50,8 \%$ dos não idosos, não havendo diferença estatisticamente significativa. A frequiência de hipertensão arterial sistêmica foi de $39,3 \%$ nos idosos e de 26,2\% em não idosos (Figura 2), porém sem significância estatística.

A forma clínica cardíaca foi a mais comum em ambos os grupos: $88,5 \%$ entre os idosos e $78,7 \%$ entre os não idosos e a digestiva ocorreu em $36,1 \%$ dos idosos e em $32,8 \%$ dos não idosos, sem haver diferenças estatisticamente significativas. A forma indeterminada foi verificada em 1,6\% dos doentes do Grupo 1 e em 18\% daqueles do Grupo 2, havendo diferença estatisticamente significativa (Figura 3).

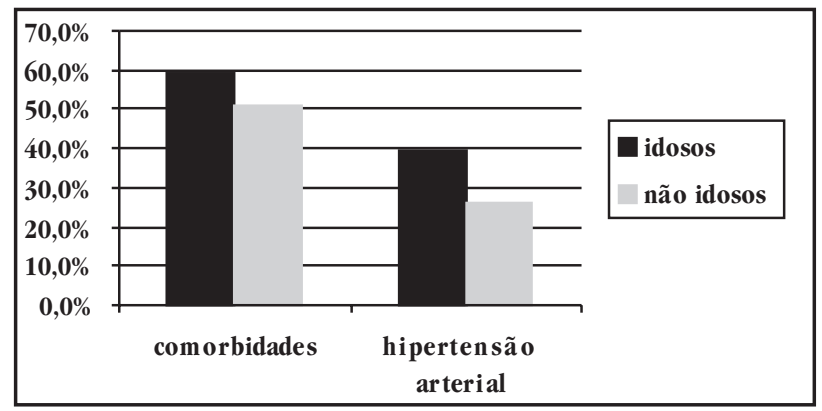

Figura 2 - Frequiência de comorbidades nos individuos dos grupos 1 e $2(p>0,05)$.

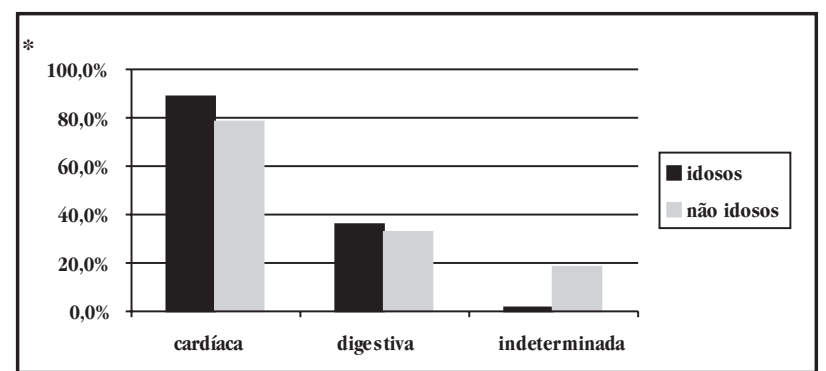

Figura 3 - Formas clínicas nos indivíduos do grupo 1 e 2 (p< 0,05 para a forma indeterminada).

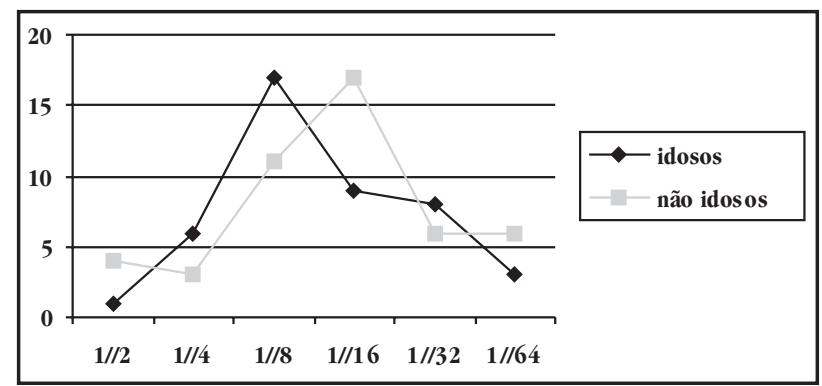

Figura 4 - Títulos da reação sorológica fixação de complemento.

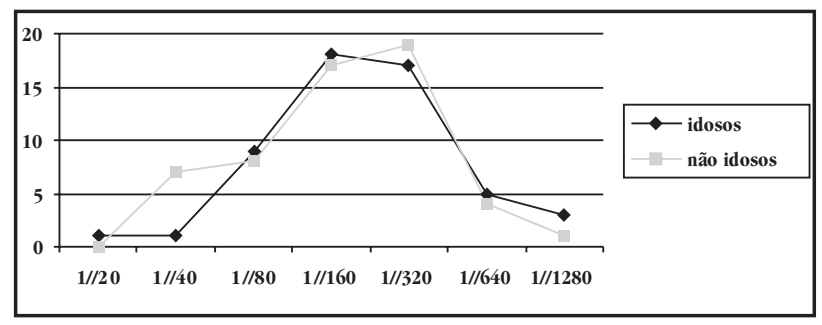

Figura 5 - Títulos da reação sorológica imunofluorescência indireta.
Com relação ao eletrocardiograma, no grupo dos idosos houve $52(82,2 \%)$ dos traçados com anormalidades e 41 (67,2\%) de anormais em não idosos. As três alterações mais frequientes em ambos os grupos foram: bloqueio divisional ântero superior esquerdo, bloqueio do ramo direito e extrassístoles ventriculares (Tabela 2). No entanto, apesar de apresentarem frequiência superior no grupo de idosos, não houve diferença estatisticamente significante.

Os títulos das sorologias para a doença de Chagas são mostrados nas Figuras 4 e 5, respectivamente. A análise destes gráficos mostra grande semelhança entre os dois grupos, não ocorrendo diferença estatisticamente significante.

\section{DISCUSSÃO}

A falta de investigações sobre a doença de Chagas em indivíduos idosos contrasta com a importância da enfermidade nessa faixa etária para a compreensão de muitos pontos ainda não devidamente esclarecidos ${ }^{9}$. 0 aumento da média de idade dos indivíduos com a doença de Chagas pôde ser esperado com a interrupção da transmissão vetorial, comprovando o sucesso das campanhas de controle vetorial e, tendo em vista a melhoria da abordagem diagnóstica e terapêutica, tornam-se prioritários os estudos sobre os aspectos clínicos da moléstia de Chagas na faixa de idade geriátrica ${ }^{14}$.

Considera idoso todo indivíduo a partir de 60 anos, mas, em nações desenvolvidas, onde a expectativa de vida é maior, o limite é estendido aos 65 anos $^{12}{ }^{18}$. Neste estudo, assim como outros que abordaram a doença de Chagas no idoso $0^{5820}$, adotou-se como limite inferior aquele preconizado em censo brasileiro de $2002^{28}$ a idade de 60 anos, embora exista, ainda, uma subclassificação entre idosos jovens ( 60 a 74 anos), idosos velhos ( 75 a 84 anos) e idosos muito velhos (mais de 85 anos).

A média de idade do grupo de chagásicos idosos $(66,03$ anos) encaixa-se na subclassificação de idosos jovens, dentro da classificação já citada, a qual corresponde à faixa com o maior número de idosos no Brasil ${ }^{28}$. Na casuística em discussão, não houve nenhum doente da subclassificação idoso muito velho, o que ocorreu em outro estudo sobre o assunto, onde foram encontrados 10 chagásicos com mais de 90 anos $^{8}$. Não foram encontrados, também, doentes com menos de 22 anos de idade, fato constatado na literatura onde em 496 casos de megacólon e megaesôfago não foram encontrados indivíduos abaixo dos 20 anos de idade atribuindo-se esta observação a uma efetiva interrupção da transmissão vetorial ${ }^{19}$, o que está sendo comprovado em inquéritos epidemiológicos os quais vêm demonstrando a progressiva redução da soropositividade nas faixas etárias mais jovens ${ }^{14}{ }^{26}$. No presente estudo, a média de idade entre os não idosos de 39,30 anos encontra-se em acordo com as observações encontradas na literatura ${ }^{15}$.

A predominância da doença de Chagas em determinado sexo já tem sido motivo de estudo, mostrando-se controverso, uma vez que alguns têm encontrado predomínio de indivíduos do sexo masculino5. Já em outros o risco seria igual para ambos 
os sexos ${ }^{6}$. Encontrou-se no presente estudo maior número de indivíduos do sexo feminino, tanto no grupo de chagásicos idosos $(67,2 \%)$, quanto no grupo de não idosos $(54,1 \%)$, porém sem haver diferença estatística. Este dado encontra-se em acordo com outros da literatura ${ }^{89} 26$ os quais, também, não observaram diferenças significativas na freqüência de homens e mulheres entre chagásicos, analisando-se todas as faixas etárias. Assim, não parece razoável atribuir sobrevida maior, nem predominância da doença em um dos sexos.

0 grupo de doentes idosos apresentou cifra de 39,3\% de hipertensos, sendo a única comorbidade relevante na casuística em discussão. Frequiência elevada desta comorbidade tem sido relatada em outros estudos, inclusive avaliando a hipertensão arterial em indivíduos idosos portadores de doença de Chagas que morreram e foram autopsiados, nos quais a cardiopatia por hipertensão atingiu a freqüiência de $37 \%$, sendo esta bem superior às outras cardiopatias encontradas, tais como isquêmica, secundária à doença pulmonar, valvopatias entre outras ${ }^{921}$. Na série do presente estudo esta frequiência de hipertensão arterial seria de $40,7 \%$, se fossem considerados apenas os doentes da forma cardíaca da doença de Chagas.

A possível influência da hipertensão arterial em idosos em relação à história natural da doença de Chagas e vice-versa tem sido objeto de estudo o qual analisou variáveis da função cardíaca e não atribuiu à hipertensão condição agravante ou determinante de disfunção cardiovascular nestes indivíduos ${ }^{11}$. No entanto, outros autores ${ }^{1617}$ referem que 50\% dos indivíduos com a associação da doença de Chagas e hipertensão arterial sistêmica têm mais de 45 anos e algum grau de insuficiência cardíaca, atribuindo à fisiopatologia da tripanossomíase a condição de fator contribuinte para a elevação da pressão arterial. Os resultados do presente estudo, entretanto, não confirmam estes últimos achados, pois não houve diferença significativa entre o grupo de idosos e não idosos em relação à frequiência de hipertensão arterial sistêmica. A hipertensão arterial, provavelmente, manifesta-se com o avançar da idade independentemente da evolução da doença de Chagas. Todavia, outros estudos são necessários para esclarecer estes dados controversos relacionados a este tema.

A forma clínica predominante nos dois grupos foi a forma cardíaca, seguida da digestiva e indeterminada. Outros autores ${ }^{9}$ encontraram predominância da forma digestiva em doentes idosos $(65,5 \%)$, seguida da cardíaca $(18,2 \%)$ e da indeterminada (16,2\%). Atribuíram esses dados à morte mais precoce dos portadores da cardiopatia chagásica. Este fato tem sido verificado na literatura médica sobre a doença de Chagas em relação aos doentes não idosos, nos quais a cardiopatia chagásica é a maior responsável por morte. Porém, em chagásicos idosos as causas de morte são semelhantes às de idosos não chagásicos ${ }^{320}$.

No presente estudo, tanto a forma cardíaca como a digestiva tiveram freqüência semelhante em idosos e não idosos. Tal fato pode encontrar explicação na freqüência da forma indeterminada, que foi estatisticamente superior no grupo de chagásicos com menos de 60 anos. Tais indivíduos, cuja grande maioria encontrase na quarta década de vida, apenas com sorologia reagente para a doença de Chagas, tendem a manifestar sua doença clinicamente com o passar dos anos, justificando a baixa frequiência da forma indeterminada em chagásicos idosos. Assim, ao superar os 50 anos de idade sem cardiopatia grave, conforme já verificado por outros autores ${ }^{8}$, a sobrevida não é sensivelmente afetada pela doença de Chagas. 0 estudo em discussão sugere que grande parte desses indivíduos na forma indeterminada evolua para a forma cardíaca, compensando o número de não idosos com cardiopatia chagásica que morre precocemente, mantendo elevada a freqüência da forma crônica cardíaca, mesmo entre os idosos.

As anormalidades mais comuns verificadas ao eletrocardiograma foram o BDASE, seguido do BRD e de EV; tais alterações sugestivas da cardiopatia chagásica crônica, não sendo encontradas, neste estudo, diferenças estatisticamente significativas entre os eletrocardiogramas dos chagásicos idosos e dos não idosos. Esses dados reforçam a hipótese de que a cardiopatia apresentada pelos idosos estudados se deva, ainda que talvez não exclusivamente, também à cardiopatia chagásica e não somente a outras cardiopatias habitualmente incidentes nesta faixa etária.

Com relação aos títulos das sorologias, as curvas mostradas nas Figuras 4 e 5 são muito semelhantes, não se evidenciando grandes diferenças entre idosos e não idosos. Os valores $1 / 8 \mathrm{e}$ 1/16 são os mais frequientes para a fixação de complemento. Já as curvas da imunofluorescência indireta quase que se sobrepõem, não se constituindo, assim, a sorologia convencional para o diagnóstico da doença de Chagas, um marcador para a fase em que a doença se encontra. Todavia, a manutenção dos títulos das sorologias positivos em idosos, pode ser uma prova de que o T. cruzi encontra-se presente, induzindo à formação de anticorpos por tempo prolongado, provavelmente originando novas lesões e sendo responsável pelo caráter evolutivo da doença. Este fato, entre outros, permite inferir que o tratamento específico para o parasito tenha indicação, também, para os idosos, não se restringindo a mesma às crianças e adultos jovens como propõe a literatura ${ }^{7}$.

Assim, o presente estudo permite concluir que a doença de Chagas em idosos não possui apresentação clínica diferente da doença em não idosos, justificando a investigação diagnóstica em todos os indivíduos com idade superior a 60 anos com epidemiologia ou quadro clínico sugestivo para esta doença. Também, que a forma clínica indeterminada ao ser mais freqüente em não idosos sugere o caráter evolutivo da doença.

\section{REFERÊNCIAS}

1. Akhavan D. Analise de custo-efetividade do programa de controle da doença de Chagas no Brasil, relatório final, Organização Pan-Americana da Saúde, Brasília, 2000.

2. Almeida EA, Curti HJV, Couto OC, Oliveira MAP, Santos MAL, Carvalhal SS. Doença de Chagas em pacientes acima de 60 anos. Análise de 10 casos necropsiados com ênfase à cardiopatia. In: Anais da II Reunião Anual em Pesquisa Aplicada em Doença de Chagas, Araxá, p. 34, 1985.

3. Almeida Netto JC, Mello JV, Barbosa W. Doença de Chagas: correlação sorológica e eletrocardiográfica em um grupo de indivíduos idosos. Revista da Sociedade Brasileira de Medicina Tropical 4: 75-81, 1970. 
4. Barreto M, Andrade MEB. Impacto da infecção chagásica sobre algumas características demográficas. Resultados de um estudo ecológico. Cadernos de Saúde Pública 10: 273-280, 1994.

5. Brabin $\mathrm{L}$. The epidemiological significance of Chagas'disease in women. Memórias do Instituto Oswaldo Cruz 87: 73-79, 1992.

6. Camargo ME, Silva GR, Castilho EA, Silveira AC. Inquérito sorológico de prevalência da infecção chagásica no Brasil-1975/1980. Revista do Instituto de Medicina Tropical de São Paulo 26: 192-204, 1984.

7. Cançado JR. Terapêutica específica. In: Dias JCP, Coura JR (eds) Clínica e Terapêutica da doença de Chagas. Uma abordagem prática para o clínico geral, $1^{a}$ edição, Fundação Oswaldo Cruz, Rio de Janeiro, p. 323-351, 1997.

8. Carneiro 0, Rezende JM. Doença de Chagas e Longevidade. Arquivos Brasileiros de Cardiologia 38: 381-384, 1982.

9. Carvalho Filho ET, Figueira JC, Pasini U, Forti NA, Curiati JA, Ferreira MC, Azul LG. Aspectos da doença de Chagas no idoso. Arquivos Brasileiros de Cardiologia 45: 103-107, 1985

10. Chagas C. Nova tripanozomose humana. Estudos sobre a morfologia e o evolutivo do Schizotrypanum cruzi n.gen, n.sp., agente etiológico de nova entidade mórbida do homem. Memórias do Instituto Oswaldo Cruz 1: 155-219, 1909.

11. Correia PC, Ribeiro ALP, Rocha MOC. Influência da hipertensão arterial na determinação de alterações funcionais cardiovasculares em pacientes chagásicos idosos. Revista da Sociedade Brasileira de Medicina Tropical 33 (supl 2):84, 2000.

12. Costa EF, Porto CC, Almeida JC, Cipullo JP, Martin JF. Semiologia do idoso. In: Porto CC (ed) Semiologia Médica, $4^{a}$ edição, Guanabara Koogan, Rio de Janeiro, p.165-197, 2001

13. Dias JCP. Doença de Chagas em Bambuí, Minas Gerais, Brasil: estudo clínico epidemiológico a partir da fase aguda entre 1940 e 1982 . Tese de Doutorado, Universidade Federal de Minas Gerais, Belo Horizonte, MG,1982.

14. Dias JCP. O Controle da doença de Chagas no Brasil. In: Silveira AC (ed) 0 controle da doença de Chagas nos países do cone sul da América. História de uma iniciativa internacional 1991/2001. Faculdade de Medicina do Triângulo Mineiro, p. 145-250, 2002.

15. Dias JCP, Coura JR. Epidemiologia. In: Dias JCP, Coura JR (eds) Clínica e Terapêutica da doença de Chagas. Uma abordagem prática para o clínico geral, $1^{\text {a }}$ edição, Fundação Oswaldo Cruz, Rio de Janeiro, p. 33-65, 1997.
16. Guariento ME, Orosz JE, Gontijo JA. Interação clínica entre moléstia de Chagas e hipertensão arterial primária em um serviço de referência ambulatorial. Arquivos Brasileiros de Cardiologia 70: 431-434, 1998.

17. Guariento ME, Ramos MC, Gontijo JA, Carvalhal SS. Doença de Chagas e hipertensão arterial primária. Arquivos Brasileiros de Cardiologia 60: 71-75, 1993.

18. Instituto Brasileiro de Geografia e Estatística-IBGE, www.ibge.gob.br, Perfil dos idosos responsáveis pelos domicílios. Brasília, 2002.

19. Menegelli UG, Ejima FH, Silva LR. Evidências do declínio da ocorrência do megaesôfago e do megacólon chagásicos: Estudo epidemiológico no Hospital das Clínicas de Ribeirão Preto. Medicina (Ribeirão Preto) 24: 218-224, 1991.

20. Menezes M, Rocha A, Silva AC, Silva AM. Causas básicas de morte em chagásicos idosos. Arquivos Brasileiros de Cardiologia 52: 75-78, 1989.

21. Oliveira FA, Reis MA, Teixeira PA. A cardiopatia chagásica em idosos necropsiados. Revista da Sociedade Brasileira de Medicina Tropical 34 (supl 3): 161-162, 2001.

22. Oganização Mundial da Saúde(OMS)/Organização Pan-Americana da Saúde (OPAS) Certificação do Brasil como área interrompida de transmissão da doença de chagas pelo T. infestans. Relatório Técnico do Ministério da Saúde do Brasil, 2006.

23. Paula ASV. Levantamento da mortalidade e aposentadoria por Doença de Chagas nas Regiões de Minas Gerais. In: Décourt LV, Campos OM (eds) Modernos conhecimentos sobre Doença de Chagas, $1^{\mathrm{a}}$ edição, Universidade Federal de Minas Gerais, Belo Horizonte, p. 308-314, 1981.

24. Prata A. Evolution of the clinical and epidemiological knowledge about Chagas'disease 90 years after its discovery. Memórias do Instituto Oswado Cruz 94:81-88, 1999.

25. Siqueira-Batista R, Rubião ECN, Cotta RMM, Pissinatti A, Soranz DR, Silva Pinto LF. Epidemiologia e Ecologia. In: Siqueira-Batista R, Gomes AP, Corrêa AD, Geller M (eds) Moléstia de Chagas, $2^{a}$ edição, Rubio, Rio de Janeiro, p. 147-168, 2007.

26. Souza AG, Wanderley DMV, Buralli G, Andrade JCR. Consolidation of the control of Chagas'disease vector in the state of São Paulo. Memórias do Instituto Oswaldo Cruz 79: 125-132, 1984

27. Wendell S. Doença de Chagas transfusional. In: Dias JCP, Coura JR (eds) Clínica e terapêutica da doença de Chagas: Uma abordagem prática para o clínico geral, $1^{\mathrm{a}}$ edição, Fundação Oswaldo Cruz, Rio de Janeiro, p. 411-422, 1997.

28. World Health Organization. Control of Chagas'disease - Report a WHO Expert Comeete. WHO Technical Report Series nº 811, p. 1-95, 1991. 